\title{
The method of the examination of dangerous structures and rehabilitationof their robustness
}

\author{
Avtandil Gubeladze ${ }^{1}$,Elena Yagovkina ${ }^{1, *}$, and Ivan Gubeladze ${ }^{1}$ \\ ${ }^{1}$ Don State Technical University (DSTU), Rostov-on-Don
}

\begin{abstract}
There are a variety of methods concerning the examination of dangerous structures and rehabilitation of their robustness. One of the methods is uplifting and alignment of buildings with the use of hydraulic jack. Development of the uplift and alignment program is possible if there is a data, which is to be received both before the beginning of the uplift and during the process of recovery of spatial geometry of the building. The development of the uplift and alignment program automatically controls the speed rate of the hydraulic system. The suggested method enables an accurate control of a vertical reach and provides the minimal overload on deformable footing and structure of the building during its uplift and alignment.
\end{abstract}

There are many methods that have been developed over the years to examine the dangerous structures. One of them is uplift and alignment of buildings with the use of hydraulic jack.

Depending on the type of deformation of the building, there are two possible ways of reconstructing its spatial and internal geometry: the transformation of the inclined deformation plane into a horizontal plane, or the transformation of the deformation plane into an inclined plane, and then the inclined plane into a horizontal plane.

The procedure for determining the deformationplaneis the following:when the building is being examined, the specified horizontal sections are leveled with a pitch, usually corresponding to the interaxial distances (with the pitch of the bearing building structures). At the same time, at least three given sectionsare assigned. These may include: the identified masonry belt, the lower (upper) end of the wall panel of the same floor, architectural belts made by wall masonry, the top surfaces of the foundation blocks, etc. Additional floors may include floors of the first floor (ceiling of the basement), window openings (upper or lower bound), other architectural elements of the building, the design location of which is known $[1,2]$.

The implementation of ways to restore spatial geometry also varies. There are the following operating principles of the hydraulic system:

1) the hydraulic system operates at a constant speed on all the jacks and controls the equalization process by a factor of time (with a common for all jacks pumping station);

\footnotetext{
* Corresponding author: yagovkinaalena@yandex.ru
} 
2) the system works with different jack speeds with a fixed total running time (at individual pumping stations);

3) combinedversion, when the speed of the system and the time of its operation vary (pulsating technique) (Figure 1).

Depending on the two above described factors, a rehabilitation program is assigned and then its methodology is developed. Whereas, the development of the whole complex of works on uplifting and alignment of buildings is not possible without the basic data, as well as data obtained directly in the process of reconstructing the spatial geometry of the structure [3].

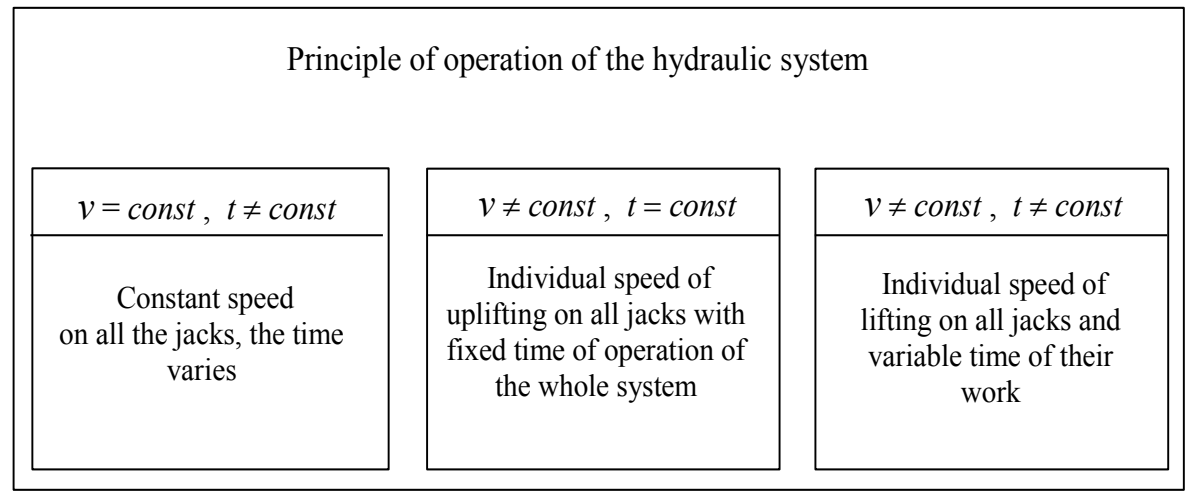

Fig. 1. The diagram of the operating principles of the hydraulic system

Currently, there are methods for monitoring the geometric parameters of a building using inclinometers, total stations and scanners. However, they all have a few drawbacks, such as: the high cost of equipment, the survey of the object between the uplifting cycles, as well as a long time spent processing and digitizing the data obtained, respectively.

As an innovation, a technology based on the operation of a hardware-system complex can be considered. This technology includes a software and computer complex, a system of hydraulic jacks with electro valves, load and displacement sensors and two robotic total stations (Figure 2).

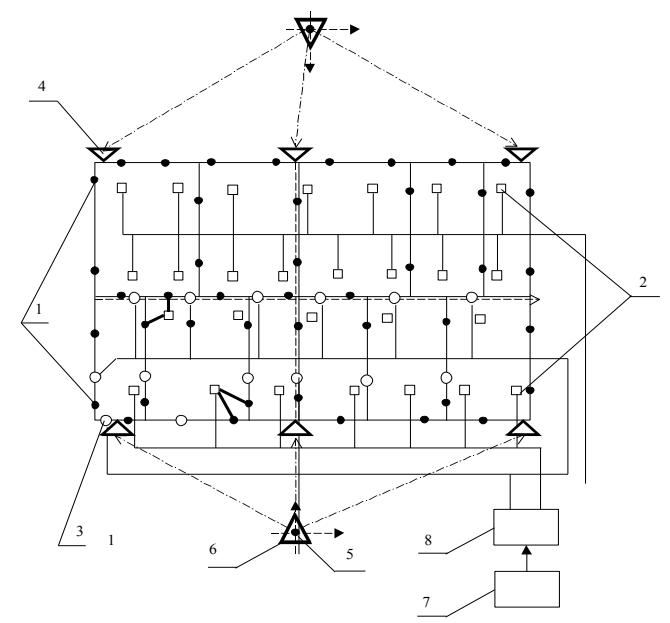

Fig. 2. Scheme of the automated system of uplifting and alignment buildings

1 - flat jacks; 2 - pumping stations; 3-resistance-type transducers; 4 - reflectors; 5 - the points of geodetic control; 6 - robotic total stations; 7 - computer; 8 - control unit. 
Total stations can be installed both from two opposite sides of the building (Figure 3), and from two cross-lying corners, depending on the situation.

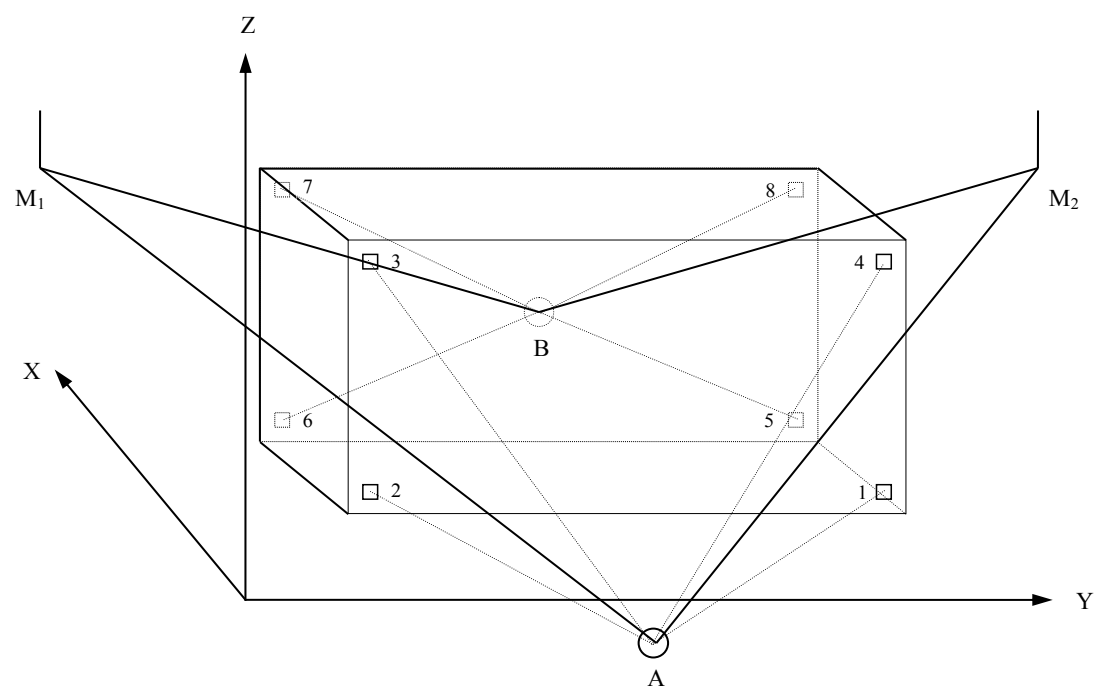

Fig. 3. Scheme of observations of points using electronic total stations

- conventional symbol for the point of standing of the instrument; 1- numbers of reflectors

A robotic total station is usually installed in a place not subject to deformation, from which a good view of the observed object is provided. If it is impossible to ensure stability of the installation site, it can place several reference reflectors in stable zones, and the total station will periodically determine its position by resection using these reflectors.

In the locations of control points highly-precise reflectors are installed. Theyare securely fixed to the object. The offset of the control point causes the reflector shifting. The total station, using the control software, determines the location of the reflectors in the local coordinate system [4].

After installing the total stations at the stations and bringing them into the working position, your own coordinate systems of the total stations should be corresponded to the coordinate system of the building. First, the coordinates of all control points of the building are plotted. During the uplift, the coordinates of the same monitored points are plotted at certain intervals.

Measurements can be taken both directly from a tripod, and from stationary base stations. In front of the building one or several observation stations are fixed, the number of which depends on the size of the building. In the second case, stationary concrete or metal poles of a special design, on which the centers are fixed,are mountedin front of the building in convenient for observation locations. The points from which measurements are taken are included in the geodetic base. All measurements are taken in a single coordinate system, and for the convenience it is reasonable to observe in the coordinate system of the building itself.

The use of two electronic total stations in controlling the process of uplift and alignment a building is reduced to a continuous, sequential determination of the coordinates $x_{i}, y_{i}, z_{i} \mathrm{of}$ the corresponding control points $[5,6]$.

By the difference in the coordinates obtained before and during the alignment process, the longitudinal and transverse relative inclinations of the vertical edges of the building the relative angles of twisting of the edges and planes of the walls, the torsional angles of the 
building and its parts, the horizontal displacement of the object, and the relative elongation of the longitudinal dimensions of the walls are calculated.

The most informative about the alignment process are the relative longitudinal and transverse inclinations of the vertical edges of the building.

They are calculated by formulas for each edge of the building fixed by the researched points:

$$
\begin{gathered}
q_{x \text { otn }}=\frac{\left(x_{i}-x_{i}^{0}\right)-\left(x_{j}-x_{j}^{0}\right)}{\left[\left(x_{i}-x_{j}\right)^{2}+\left(y_{i}-y_{j}\right)^{2}+\left(z_{i}-z_{j}\right)^{2}\right]^{1 / 2}}, \\
q_{y \text { otn }}=\frac{\left(y_{i}-y_{i}^{0}\right)-\left(y_{j}-y_{j}^{0}\right)}{\left[\left(x_{i}-x_{j}\right)^{2}+\left(y_{i}-y_{j}\right)^{2}+\left(z_{i}-z_{j}\right)^{2}\right]^{1 / 2}},
\end{gathered}
$$

where $\mathrm{i}, \mathrm{j}$ - the numbers of the points forming the edge of the structure; $x_{i, j}^{0}, y_{i, j}^{0}, z_{i, j}^{0}{ }_{-}$coordinates of the corresponding points, determined before alignment; $x_{i, j}, y_{i, j}, z_{i, j}$ the coordinates of the corresponding points, determined during the alignment process.

Receiving coordinates of the corners of the building faces, one can make conclusions considering the processes taking place with them. So, for example, changing the difference in the coordinates $x_{i}$ of the researched points, located in one horizontal section, gives us information about the compression of the wall. It should be noted that the use of reflectorless total stations allows to plot the coordinates in any fixed points of a building. In this case, the solution to the problem of finding geometric parameters is simplified, since it becomes possible to obtain the coordinates of those points of the building in which it is not possible to install marks with reflectors. When the edges of the building edges reach the design point, the uplift program informs the operator about the completion of the process [3].

Also, in the process of uplifting, simultaneously with plotting of the coordinates of the control points of the corners of the building, time is recorded. When processing the received data, one receives additional information about the processes occurring with the building. In this case, the diagram of the interdependence of the inclinations, the compression of the walls, the angles of the twisting of the walls and the values of the uplifting from time, are created.

The coordinates $(\mathrm{x}, \mathrm{y}, \mathrm{z})$ of the points fixed on the building are plotted in a certain period of time, $\mathrm{t}$. The observation period $t_{0}=0$ is taken as the initial one and it corresponds to the coordinates of the points $1,2,3$, and 4 , i.e. $x_{i}^{0}, y_{i}^{0}, z_{i}^{0}$. It is assumed here that the initial velocities of the displacement of these points are: $v_{x_{i}}^{0}=0 ; v_{y_{i}}^{0}=0 ; v_{z_{i}}^{0}=0$.

In the timeperiod $t_{1} \neq 0$, the spatial coordinates of points $1,2,3$ and $4\left(x_{i}^{1}, y_{i}^{1}, z_{i}^{1}\right)$ are plotted and coordinate increments are found

$$
\Delta x_{i}^{0-1}=x_{i}^{1}-x_{i}^{0} ; \Delta y_{i}^{0-1}=y_{i}^{1}-y_{i}^{0} ; \Delta z_{i}^{0-1}=z_{i}^{1}-z_{i}^{0} .
$$

Correspondingly, the velocities of displacement of points in time $\Delta t_{1}=t_{1}-t_{0}$ are:

$$
v_{x_{i}}^{1}=\frac{x_{i}^{1}-x_{i}^{0}}{t_{1}} ; v_{y_{i}}^{1}=\frac{y_{i}^{1}-y_{i}^{0}}{t_{1}} ; v_{z_{i}}^{1}=\frac{z_{i}^{1}-z_{i}^{0}}{t_{1}} .
$$

Over the followingperiod of time, the values of the change in the velocities of the displacement of points 1, 2, 3, and 4 are determined: 


$$
a_{x_{i}}^{1}=\frac{v_{x_{2}}-v_{x_{1}}}{\Delta t^{1-2}} ; a_{y_{i}}^{1}=\frac{v_{y_{2}}-v_{y_{1}}}{\Delta t^{1-2}} ; a_{z_{i}}^{1}=\frac{v_{z_{2}}-v_{z_{1}}}{\Delta t^{1-2}} .
$$

Hereafter, one is given a certain time interval $\Delta t_{3}^{T}$ and one calculates the approximating function of the values of the speeds to which each point should move for the time period, i.e. $v_{x_{3}}^{T}, v_{y_{3}}^{T}, v_{z_{3}}^{T}$ and correspondingly $a_{x_{3}}^{T}, a_{y_{3}}^{T}, a_{z_{3}}^{T}$, which will allow to determine the expected spatial position of the researched points.

According to the established time interval $t_{3}$, measurements are taken and the coordinates of points $1,2,3,4 . x_{i}^{3}, y_{i}^{3}, z_{i}^{3}$.

Hereafter,the increment of coordinates that occurred over the time interval $t_{3}$ is calculated:

$$
\Delta x_{i}^{2-3}=x_{i}^{3}-x_{i}^{2} ; \Delta y_{i}^{2-3}=y_{i}^{3}-y_{i}^{2} ; \Delta z_{i}^{2-3}=z_{i}^{3}-z_{i}^{2} .
$$

The speed and acceleration of moving marks is determined. Analyzing the obtained dependencies, we can predict the state of the building at the following period of time. When the actual data are received at the certain time point, the results are compared, and the uplifting process mode is adjusted. Based on the measurement results, all parameters characterizing the state of the building are calculated and saved in the database.

After determining all the necessary data, the program sends them to the control unit. In a moment, an automatically updated warping of the building being lifted, with significantly enlarged banks of the edges of the given structure, is presented on the monitor screen for a better picture of the state of the building being lifted. There are also windows for working with the database, in which (as the data arrives) records are automatically added.

In the process of controlling the uplift and alignment of the building, the instruments and control points are arranged according to a pre-selected scheme (Figure 4). In the process of uplifting, the measurements were taken using a batter level with bar-code rails and an electronic total station (three-dimensional coordinates of the monitored points), all measurements were timed. The results of the obtained values are presented in Figure 1 to 3.

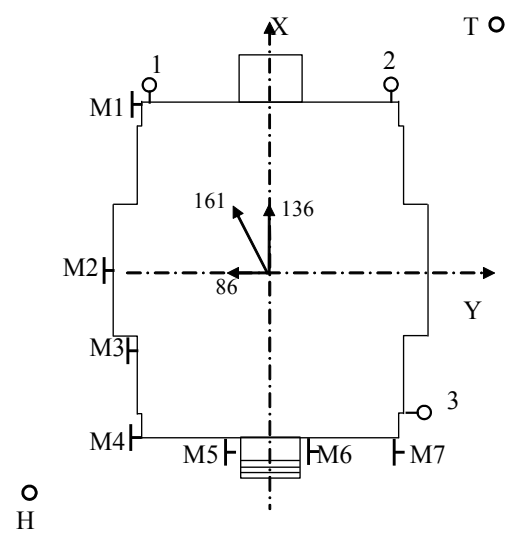

Fig. 4. Arrangement of marks of leveling and reflectors on the building when uplifting

H- location of the electronic level; T- location of the electronic total station, M1-M7-lined rods; 1, 2film reflectors; 3-prismatic reflectors (reflectors are located at the top and bottom of the building) 
Table 1. The values of vertical displacements ascending by $X$

\begin{tabular}{|c|c|c|c|c|c|c|c|c|}
\hline Time & $10: 10$ & $10: 20$ & $10: 30$ & $10: 40$ & $10: 50$ & $11: 00$ & $11: 10$ & $11: 20$ \\
\hline Mark 1 & 0,0 & 0,0 & 5,5 & 12,4 & 22,1 & 30,8 & 36,8 & 39,9 \\
\hline Mark 2 & 0,0 & 0,0 & 9,2 & 17,0 & 25,0 & 33,1 & 40,5 & 42,1 \\
\hline Mark 3 & 0,0 & 0,0 & 9,1 & 16,0 & 23,0 & 30,0 & 36,1 & 39,0 \\
\hline Mark 4 & 0,0 & 0,0 & 7,5 & 16,8 & 22,5 & 28,3 & 33,0 & 37,5 \\
\hline
\end{tabular}

Table 2. Vertical displacements along the $\mathrm{Y}$ axis

\begin{tabular}{|c|c|c|c|c|c|c|c|c|c|}
\hline $\begin{array}{c}\text { Time } \\
\text { Mark }\end{array}$ & $12: 50$ & $13: 00$ & $13: 10$ & $13: 20$ & $13: 30$ & $13: 40$ & $13: 50$ & $14: 00$ & $14: 10$ \\
\hline Mark 4 & 0,0 & 0,5 & 5,4 & 13,0 & 22,4 & 22,0 & 36,1 & 39,8 & 52,0 \\
\hline Mark 5 & 0,0 & 0,5 & 5,4 & 15,5 & 20,8 & 23,0 & 33,0 & 37,5 & 45,0 \\
\hline Mark 6 & 0,0 & 0,5 & 4,9 & 15,0 & 20,0 & 22,5 & 30,1 & 37,0 & 40,0 \\
\hline Mark 7 & 0,0 & 0,1 & 0,5 & 11,9 & 17,0 & 19,2 & 24,9 & 29,0 & 32,5 \\
\hline
\end{tabular}

Table 3. Changes in inclinations of the building edges along the $\mathrm{X}$ axis in the process recovery

\begin{tabular}{|c|c|c|c|c|c|c|c|c|c|}
\hline $\begin{array}{c}\text { Time } \\
\text { Edge }\end{array}$ & $9: 10$ & $9: 30$ & $9: 50$ & $10: 10$ & $10: 30$ & $10: 50$ & $11: 10$ & $11: 30$ & $11: 50$ \\
\hline Edge 1 & $\begin{array}{c}- \\
185,0\end{array}$ & $-180,0$ & $-168,1$ & $-150,0$ & $-129,8$ & $-112,2$ & $-88,5$ & $-69,0$ & $-49,0$ \\
\hline Edge 2 & $\begin{array}{c}- \\
132,3\end{array}$ & $-130,0$ & $-113,1$ & $-96,0$ & $-74,2$ & $-52,4$ & $-39,5$ & $-7,2$ & 0,0 \\
\hline Edge 3 & $\begin{array}{c}- \\
166,0\end{array}$ & $-155,2$ & $-151,0$ & $-127,2$ & $-93,2$ & $-76,1$ & $-60,0$ & $-30,0$ & $-28,1$ \\
\hline
\end{tabular}




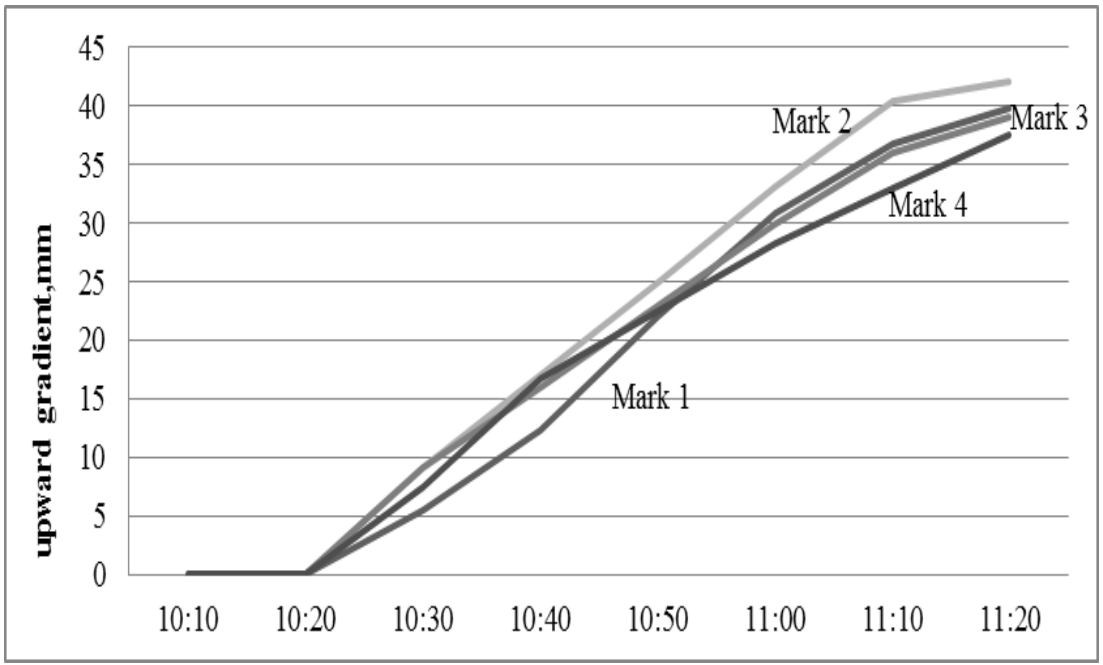

Fig. 5. The plot of the building's elevation along the $X$ axis

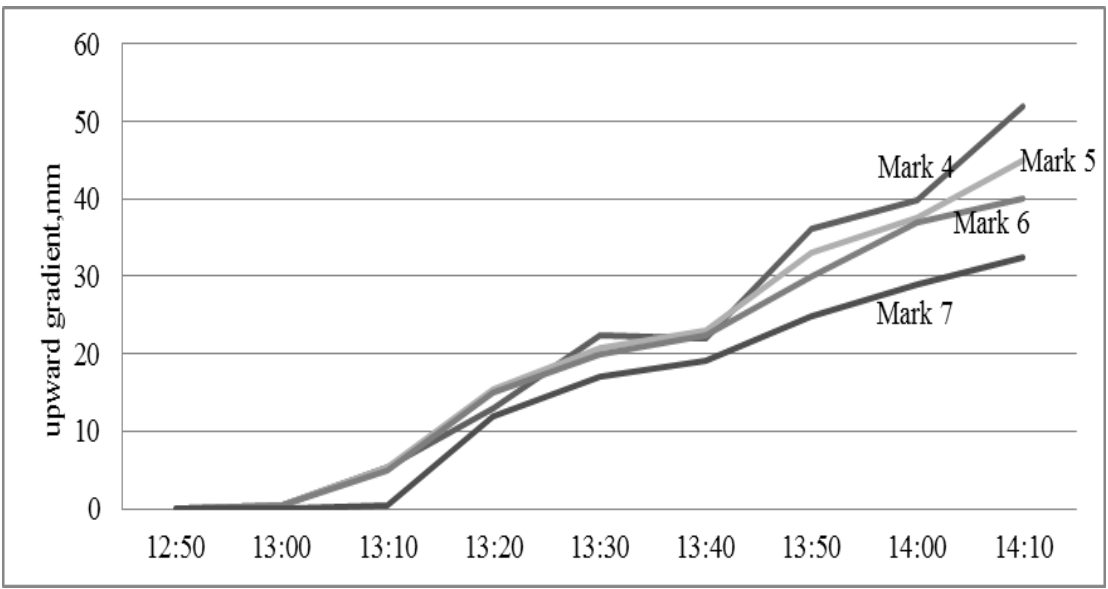

Fig. 6. Uplifting along the $\mathrm{Y}$ axis

Based on the obtained results, the diagrams of the dependence between the inclinations and the values of the uplift versus time were plotted. The building was raised with one remounting of the jack system. At the same time, the south-west corner was raised by $16 \mathrm{~cm}$. During the uplifting, the building was given a counter-inclination equal to 20 $\mathrm{mm}$. 


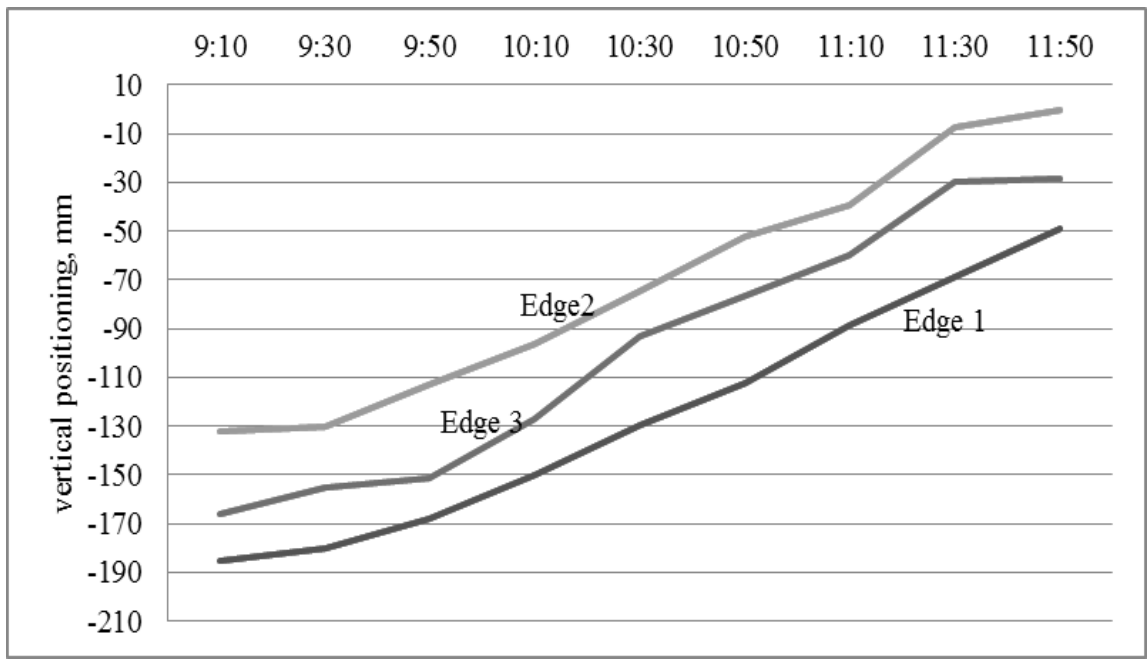

Fig. 7. Graph of changes in private heels of the building edges along the $X$ axisin the process of recovery

Thus, the building is uplifted and aligned because of the obtained values of velocities and accelerations on all jacks for a given time interval for a given uplifting cycle. This ensures an even alignment of the building without creating additional deformations orany additional destruction.

\section{References}

1. A.R. Gubeladze, I.G. Gayrabekov, Bulletin of the Union of Surveyors, 5 (2011)

2. I.G. Gayrabekov, Natural Sciences, 2 (2011)

3. A.R. Gubeladze, Applied Geodesy, RGSU, Dep. VINITI 6.12.00, no. 3091-B00, 24-31 (2000)

4. Y.I. Pimshin, M.D. Zotov, A.R. Gubeladze, Modern problems of soil mechanics and protection of the geological environment. Proceedings of the VII school-seminar, RSU (1999)

5. I.G.Gairabekov, Y.S. Zabaznov, Electronic scientific and innovation magazine, The Engineer's Herald of the Don, 4 (2010)

6. Y.I. Pimshin, A.R. Gubeladze, A.N. Bogdanov, Applied Geodesy, RGSU, Dep. VINITI 09.06.03, no. 1119-B2003, 46 (2003) 